\title{
Gastric Metastasis from a Primary Renal Leiomyosarcoma
}

\author{
Satoshi Yodonawa ${ }^{a}$ Isao Ogawa ${ }^{a}$ Susumu Yoshida ${ }^{a}$ \\ Hiromichi Ito ${ }^{a}$ Akinori Kato $^{\text {a }}$ Ryoko Kubokawa ${ }^{a}$ \\ Emika Tokoshima $^{a}$ Hiroaki Shimoyamada $^{b}$ \\ ${ }^{a}$ Department of Surgery, Ibaraki Seinan Medical Center Hospital, Sakai, and \\ ${ }^{b}$ Department of Pathology, Tsukuba University, Tsukuba, Japan
}

\section{Key Words}

Gastric metastasis · Renal leiomyosarcoma · Nephrectomy · Gastrectomy

\begin{abstract}
Primary leiomyosarcoma of the kidney is rare. Here we report a case of metastasis of this tumor to the stomach. A 73-year-old man visited our hospital suffering from general weakness and intermittent tarry stools. He had undergone right nephrectomy for renal leiomyosarcoma 2 years previously. There had been no local recurrence or distant metastasis in the 2-year follow-up period. Endoscopy revealed two submucosal tumors in the stomach. These tumors were diagnosed histologically as leiomyosarcoma and distal gastrectomy was performed. Subsequent histochemical staining confirmed the diagnosis of gastric metastasis from renal leiomyosarcoma. The patient died due to metastases to the liver and bone 9 months after the operation. To the best of our knowledge, this is the first report of gastric metastasis from primary renal leiomyosarcoma.
\end{abstract}

\section{Introduction}

Metastases to the stomach are unusual and most of them arise via a hematogenous route from malignant melanoma or carcinoma of the breast or lung [1]. Gastric metastasis from a renal tumor is rare and all reported cases involve renal cell carcinoma [2]. Leiomyosarcoma of the kidney is also uncommon, accounting for $1-2 \%$ of malignant renal tumors in adults [3], and metastasis of this tumor to the gastrointestinal tract has rarely been documented [4]. Here we report a case of primary renal leiomyosarcoma that metastasized to the stomach. 


\section{Case Report}

A 73-year-old man had undergone right nephrectomy for treatment of a right renal tumor 2 years previously. Grossly, the specimen consisted of a tumor arising at the lower pole of the right kidney. The tumor measured $12 \times 11 \times 8 \mathrm{~cm}$ and the cut surface showed the mass to be circumscribed, solid and white (fig. 1a). Histology showed that the tumor was composed of spindle cells arranged in interlacing fascicles, with elongated, hyperchromatic nuclei and eosinophilic cytoplasm. Two mitotic figures were noted per 10 high-power fields (fig. 1b). Immunostaining showed tumor cells with cytoplasm that was diffusely positive for smooth muscle actin (SMA). Based on these data, leiomyosarcoma arising from the lower pole of the right kidney was diagnosed. Gastric endoscopy before nephrectomy showed normal findings. No local recurrence or systemic metastasis was detected in the 2-year follow-up period.

The patient visited our hospital due to general weakness, epigastric pain and intermittent tarry stools 2 years after the operation. Physical examination revealed an anemic conjunctiva and abdominal tenderness in the periumbilical area. Laboratory data showed a low hemoglobin level of $4.0 \mathrm{~g} / \mathrm{dl}$ and transfusion of 4 units of blood was performed. Upper gastrointestinal endoscopy revealed two submucosal tumors: one polypoid tumor $4 \mathrm{~cm}$ in diameter located in the anterior wall of the middle body, and another tumor $3 \mathrm{~cm}$ in diameter that showed ulceration and was located in the posterior wall of the middle body (fig. 2). Histopathological examination of biopsy samples indicated a leiomyosarcoma.

Chest X-ray showed no metastases and computed tomography scans of the abdomen showed a gastric mass and several paragastric lymphadenopathies without other distant organ metastases. Therefore, distal gastrectomy was performed under a diagnosis of metastasis of renal leiomyosarcoma or gastrointestinal stromal tumor of the stomach. Subsequent histopathological examination showed spindle cells with marked pleomorphism. The number of mitotic figures was 40-50 per 10 high-power fields ( $\underline{\text { fig. } 3 \mathrm{a}}$ ). In histochemical staining, the tumor was positive for SMA (fig. 3b) and negative for c-kit (fig. 3d) and CD34 (fig. 3c), which confirmed the diagnosis of metastatic high-grade leiomyosarcoma with paragastric lymph node metastases. The postoperative course was uneventful, but the patient died due to metastases to lung, liver and bone 9 months after the operation.

\section{Discussion}

Metastases to the stomach are unusual, with the estimated incidence of gastric metastases at autopsy in patients with a known malignancy varying from 1.7 to $5.4 \%$ [5]. Up to half of patients harboring such metastases are symptomatic, most commonly with bleeding, pain, vomiting and anorexia. Hematogenous metastatic lesions usually implant in the submucosal layer of the stomach, appearing as one or multiple discrete submucosal nodules that may ulcerate, and the metastatic lesions sometimes manifest as larger masses $[5,6]$. Endoscopy may be useful for diagnosis, mainly for small nodules with or without central ulceration, polypoid masses and ulcers $[5,6]$. The common primary tumors that metastasize to the stomach are malignant melanoma and carcinoma of the breast and lung [1]. Gastric metastasis from a renal tumor is uncommon and only 15 cases have been described, all of which were renal cell carcinoma [2]. Therefore, a metastatic gastric tumor from renal leiomyosarcoma is extremely rare, and to the best of our knowledge this case is the first reported in the literature.

Leiomyosarcoma usually arises in the uterus, gastrointestinal tract and soft tissue. Primary renal leiomyosarcomas account for $1-2 \%$ of all malignancies in the adult kidney [2] and may arise from the renal capsule, renal parenchyma, pelvic musculature or main renal vein. Grossly, the tumor has the appearance of a leiomyoma with a 
well-circumscribed margin and a whorled cut surface. The malignant counterpart, however, appears fleshy and has areas of necrosis, hemorrhage and cystic degeneration [7]. Radical nephrectomy is the treatment of choice for renal leiomyosarcoma [8]. However, chemotherapy and radiotherapy are also recommended considering the aggressive behavior of the neoplasm. Renal leiomyosarcomas have rapid growth and a high metastatic tendency, and show both local and distant recurrence. These characteristics lead to a very poor prognosis with a 5-year survival rate of 29-36\%, and most patients die within 1 year of diagnosis [9].

Management of high-grade sarcoma involves a multidisciplinary approach, including surgical resection, radiotherapy and adjuvant chemotherapy. The possibility of gastric metastasis should be considered in patients who present with upper gastrointestinal bleeding [10]. Patients with bleeding from a gastric metastasis due to high-grade sarcoma often have widespread disease at presentation, and thus the role of surgery is limited. Surgical resection of a gastric metastasis may be performed to control life-threatening bleeding, but most patients do not receive a long-term benefit, as shown in the current case.
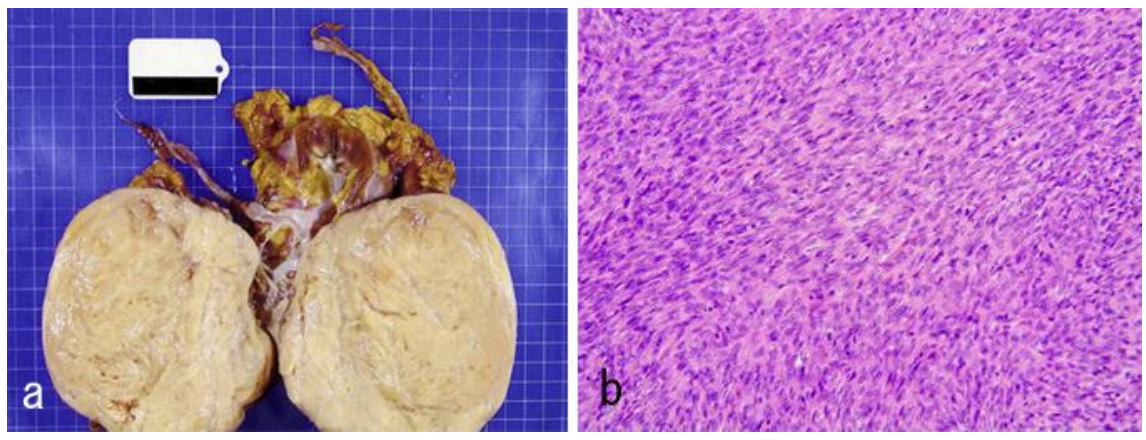

Fig. 1. a Macroscopic findings for the resected specimen. The tumor formed a circumscribed, solid, white mass at the lower pole of the right kidney. b Histopathologically, the tumor was composed of spindle cells arranged in interlacing fascicles, with elongated, hyperchromatic nuclei and eosinophilic cytoplasm. Two mitotic figures per 10 high-power fields were noted (hematoxylin-eosin staining, $\times 200$ ). 


\begin{tabular}{r|l|l|l} 
Case Reports in & $\begin{array}{l}\text { Case Rep Gastroenterol 2012;6:314-318 } \\
\text { DOI: 10.1159/000338837 }\end{array}$ & $\begin{array}{l}\text { Published online: } \\
\text { May 23, 2012 }\end{array}$ & $\begin{array}{l}\text { @ 2012 S. Karger AG, Basel } \\
\text { ISSN 1662-0631 } \\
\text { www.karger.com/crg }\end{array}$ \\
\hline
\end{tabular}
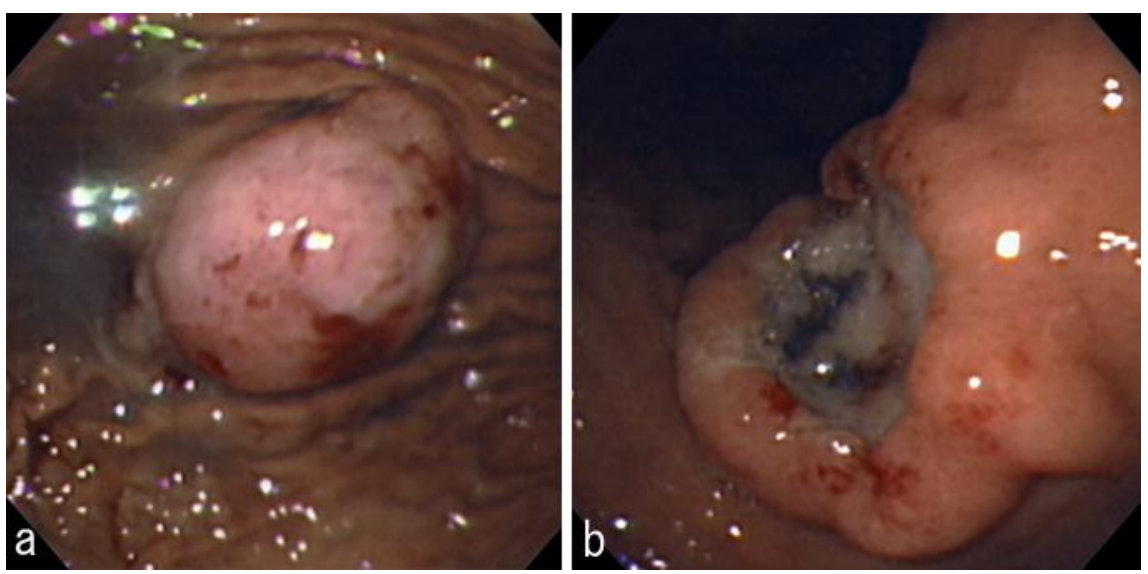

Fig. 2. Upper gastrointestinal endoscopy revealed two submucosal tumors: a polypoid tumor located at the anterior wall of the middle body (a) and an ulcerated tumor located at the posterior wall of the middle body (b).

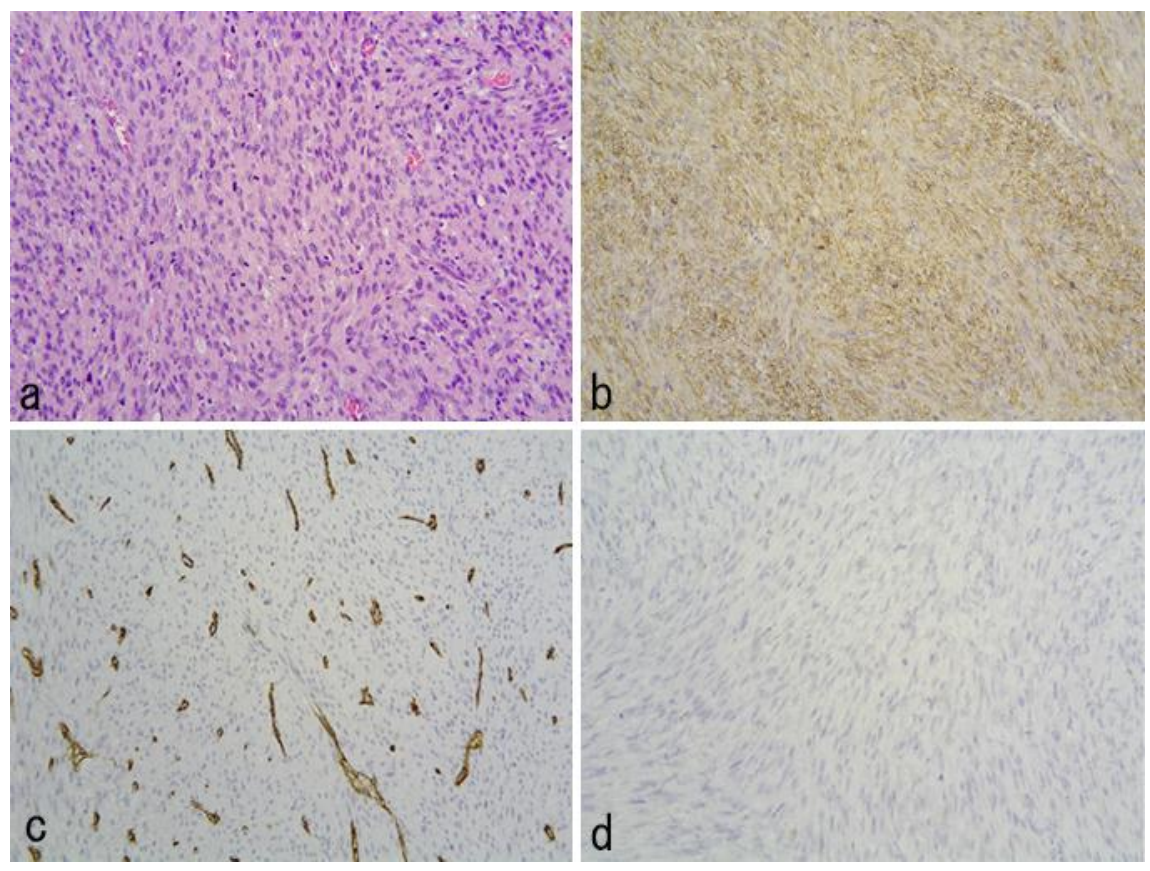

Fig. 3. Histopathological examination of the gastric tumor showed spindle cells with marked pleomorphism by hematoxylin-eosin staining $(\times 200)$. The number of mitotic figures was $40-50$ per 10 high-power fields (a). Histochemical staining of the gastric tumor was positive for SMA (b), negative for CD34 (c) and negative for c-kit (d) by immunohistological staining $(\times 200)$. 


\section{References}

1 Menuck LS, Amberg JR: Metastatic disease involving the stomach. Am J Dig Dis 1975;20:903-913.

2 Riviello C, Tanini I, Cipriani G, Pantaleo P, Nozzoli C, Poma A, Riccardo P, Valeri A: Unusual gastric and pancreatic metastatic renal cell carcinoma presentation 10 years after surgery and immunotherapy: a case report and a review of literature. World J Gastroenterol 2006;32:5234-5236.

3 Venkatesh K, Lamba Saini M, Niveditha SR, Krishnagiri C, Babu S: Primary leiomyosarcoma of the kidney. Patholog Res Int 2010;2010:652398.

-4 Buchman AL, Truong LD: Colonic metastasis from a primary renal leiomyosarcoma: an unusual cause of gastrointestinal hemorrhage. South Med J 1997;90:1238-1240.

5 Oda I, Kondo H, Yamao T, Ono H, Gotoda T, Yamaguchi H, Yoshida S, Shimoda T: Metastatic tumors to the stomach: analysis of 54 patients diagnosed at endoscopy and 347 autopsy cases. Endoscopy 2001;33: 507-510.

6 De Palma GD, Masone S, Rega M, Shimeoli I, Donisi M, Addeo P, Iannone L, Pilone V, Persico G: Metastatic tumors to the stomach: clinical and endoscopic features. World J Gastroenterol 2006;12:7326-7328.

7 Farrow GM, Harrison EG Jr, Utz DC, ReMine WH: Sarcomas and sarcomatoid and mixed malignant tumors of the kidney in adults. I. Cancer 1968;22:545-550.

-8 Demir C, Yazici CM, Eren F, Türkeri L: Case report: good prognosis in leiomyosarcoma of the kidney. Int Urol Nephrol 2007;39:7-10.

9 Dominici A, Mondani N, Nesi G, Travaglini F, Di Cello V, Rizzo M: Cystic leiomyosarcoma of the kidney. Urol Int 2000;65:229-231.

10 Dent LL, Cardona CY, Buchholz MC, Peebles R, Scott JD, Beech DJ, Ballard BR: Soft tissue sarcoma with metastasis to the stomach: a case report. World J Gastroenterol 2010;16:5130-5134. 